\title{
Energy Efficiency Maximization for UAV-Assisted Emergency Communication Networks
}

\author{
Haibin Niu $\mathbb{D}^{1}{ }^{1}$ Xinyu Zhao, ${ }^{2}$ Liming Hou, ${ }^{3}$ and Dongjun $\mathrm{Ma}^{4}$ \\ ${ }^{1}$ State Key Laboratory of Networking and Switching Technology, Beijing University of Posts and Telecommunications, \\ Beijing 100876, China \\ ${ }^{2} 27$ th Research Institute of China Electronics Technology Group Corporation (CETC), Zhengzhou 450047, China \\ ${ }^{3}$ Datang Mobile Communications Equipment Co., Ltd., Beijing 100083, China \\ ${ }^{4}$ Guangdong OPPO Mobile Telecommunications Co., Ltd., Dongguan 523860, China \\ Correspondence should be addressed to Haibin Niu; niuhaibin@bupt.edu.cn
}

Received 23 June 2021; Revised 18 July 2021; Accepted 29 July 2021; Published 18 August 2021

Academic Editor: Nagendra Kumar

Copyright (C) 2021 Haibin Niu et al. This is an open access article distributed under the Creative Commons Attribution License, which permits unrestricted use, distribution, and reproduction in any medium, provided the original work is properly cited.

Using unmanned aerial vehicles (UAVs) in emergency communications is a promising technology because of their flexible deployment, low cost, and high mobility. However, due to the limited energy of the onboard battery, the service duration of the UAV is greatly limited. In this paper, we study an emerging energy-efficient UAV emergency network, where a UAV works as an aerial base station to serve a group of users with different statistical quality-of-service (QoS) constraints in the downlink. In particular, the energy efficiency of the UAV is defined as the sum effective capacity of the downlink users divided by the energy consumption of the UAV, which includes the energy consumed by communication and the energy consumed by hovering. Then, we formulate an optimization problem to maximize the energy efficiency of the UAV by jointly optimizing the UAV's altitude, downlink transmit power, and bandwidth allocation while meeting a statistical delay QoS requirement for each user. The formulated optimization problem is a nonlinear nonconvex optimization problem of fractional programming, which is difficult to solve. In order to deal with the nonconvex optimization problem, the following two steps are used. First, we transform the fractional objective function into a tractable subtractive function. Second, we decompose the original optimization problem into three subproblems, and then, we propose an efficient iterative algorithm to obtain the energy efficiency maximization value by using the Dinkelbach method, the block coordinate descent, and the successive convex optimization technique. Extensive simulation results show that our proposed algorithm has significant energy savings compared with a benchmark scheme.

\section{Introduction}

1.1. Background and Motivation. The communication network is crucial for postdisaster rescue. However, when disasters (e.g., earthquakes and tsunamis) occur, the infrastructure of terrestrial communication networks, such as base stations, will be severely damaged [1]. Furthermore, the traditional terrestrial wireless network is difficult to be rebuilded and restore communication in a short time, because it requires a lot of equipment and manpower. Therefore, it is a huge challenge to quickly deploy an emergency network. Recently, the application of UAVs in wireless communications has attracted great attention because of their high mobility, low cost, and flexible deployment [2]. In addition, due to the mobility of the UAV, a high probability line-of-sight (LoS) link can be formed between ground users and the UAV. Thus, UAVs as aerial base stations (BSs) [3-5] and relays $[6,7]$ have been extensively studied by researchers from the scientific and industrial circles. Motivated by the above discussion, we exploit UAVs to build an emergency network to serve a group of users in a disaster area.

With the widespread use of smart devices, mobile multimedia traffic (e.g., video and remote meetings) has increased exponentially in the network. Multimedia traffic will account for more than $82 \%$ of the entire network traffic in 2021 [8]. Correspondingly, the UAV-enabled emergency network 
should provide the delay QoS guarantee for mobile multimedia traffic. Due to the time-varying nature of the wireless channel, it is difficult to provide a deterministic delay guarantee for the transmitted multimedia traffic. Thanks to the effective capacity (EC) [9-12] that it has been widely used to provide statistical delay QoS guarantee for multimedia traffic in wireless networks.

When a UAV is used as an aerial BS to provide emergency services, how to extend the service time of the UAV is an important issue, because the onboard battery of the UAV has limited energy. According to References [13, 14], the energy consumption of the UAV is related to its hovering altitude. Therefore, by carefully designing the hovering altitude and resource allocation of the UAV, the energy consumption of the UAV can be reduced when transmitting the same amount of data. Inspired by this reason, in this paper, we consider a UAV as an aerial BS to serve a group of ground users with different statistical QoS constraints in the downlink. Our goal is to maximize the energy efficiency of the UAV by jointly optimizing the altitude and resource allocation of the UAV while satisfying a statistical QoS requirement for each user.

1.2. Related Works. The works in [15-17] investigated the energy efficiency of UAVs as base stations, but the propulsion energy consumption of UAVs was ignored. The authors of [15] proposed to minimize UAV transmission power to achieve energy-efficient deployment while meeting the wireless coverage of ground users. In [16], the authors considered multiple UAVs energy-efficient three-dimensional (3D) deployment scenarios by optimizing the total transmit power to the minimum and ensuring the QoS requirement of each user. In [17], the energy efficiency of UAV-aided 5G systems with interference-aware was maximized when supporting device-to-device communication. However, since the energy consumed by UAV propulsion is usually much higher than the energy consumed by communication, optimizing the consumption of propulsion energy directly prolongs the service duration of the UAV. Therefore, the propulsion energy consumption of the UAV cannot be ignored when studying the energy efficiency of the UAV. Motivated by this reason, the propulsion energy consumptions of fixed-wing and rotary-wing UAVs were studied in detail in $[18,19]$, respectively. Further, the energy consumption models of these two UAVs are given when they are used in wireless communication. In [20], the authors studied how to maximize the energy efficiency of the UAV based on the energy consumption model of the rotary-wing UAV in [19] when the UAV was used for secure communication. Although these works [1820] consider the propulsion energy consumption of the UAV, they only focus on the energy consumption of UAVs at a fixed altitude. We can learn from $[13,14]$ that the propulsion energy consumption of the UAV is related to the flight altitude, and energy consumption can be significantly reduced by optimizing the flight altitude of the UAV. Please note that although the hovering altitude of the UAV is optimized in works [15-17], the authors only focus on improving the probability of LoS without considering the relationship with the propulsion energy consumption of the UAV. In addition, all of the above works do not consider the impact of the UAV bandwidth allocation on energy efficiency. In [21], the author studied the problem of maximizing UAV energy efficiency by jointly optimizing user division, transmit power, bandwidth allocation, and UAV trajectory.

In UAV-enabled emergency networks, besides energyefficient deployment, it is also very important to ensure the delay QoS requirement of each user. There are some previous research works $[4,22,23]$ taking into account delay in UAV-enabled networks. The authors of [4] solved the minimum delay base station placement problem under constraints of UAV energy and speed. In [22], on the premise of ensuring the QoS of delay-sensitive users, the capacity of delay-tolerant users was increased to maximize the throughput of the entire network by optimizing resource allocation and UAV's trajectory. In [23], the authors studied the optimal placement and distribution of cooperative UAVs to obtain the optimization of the overall network delays. Although the delay is considered in works $[4,22,23]$, the delay-bounding is determined. Due to the wireless channel has inherent time-varying characteristics, it is very difficult and impractical to provide a deterministic delay in UAV-enabled wireless networks. To solve this problem, the effective capacity has been extensively introduced into UAV networks to provide the statistical delay QoS provisioning for multimedia traffic users in [24-26]. The number of connectable Internet of Things (IoT) devices is maximized on the uplink of the UAV relay network, while meeting heterogeneous statistical QoS requirements of IoT devices in [24]. The authors of [25] maximized the sum effective capacity of the system by jointly optimizing the 3D location of the UAV and resource allocation under the constraint of the statistical delay QoS for each user. In [17], the effective capacity was maximized under the average power and peak rate constraints, which meet various statistical QoS requirements. However, none of the works [23-26] studies the energy-efficient deployment of the UAV.

1.3. Contributions. In this paper, we jointly consider the influence of the UAV altitude, bandwidth, and power allocation, as well as time-varying channels on the UAV energy consumption. For this reason, we propose to maximize the energy efficiency of the UAV by jointly optimizing the UAV altitude, downlink transmit power, and bandwidth allocation while meeting a statistical delay QoS requirement for each user. The main contributions of this paper are given as follows:

(i) In this paper, we consider a UAV with limited energy as a base station used in a disaster area where it lacks ground facilities. When we establish the UAV energy consumption model, we consider that the energy consumption is related to the hovering altitude. Because the time-varying characteristics of the wireless channel cause uncertain delay boundaries for data transmission, we adopt the effective capacity theory to establish a statistical delay QoS guarantee. Besides, we apply the LoS probability- 


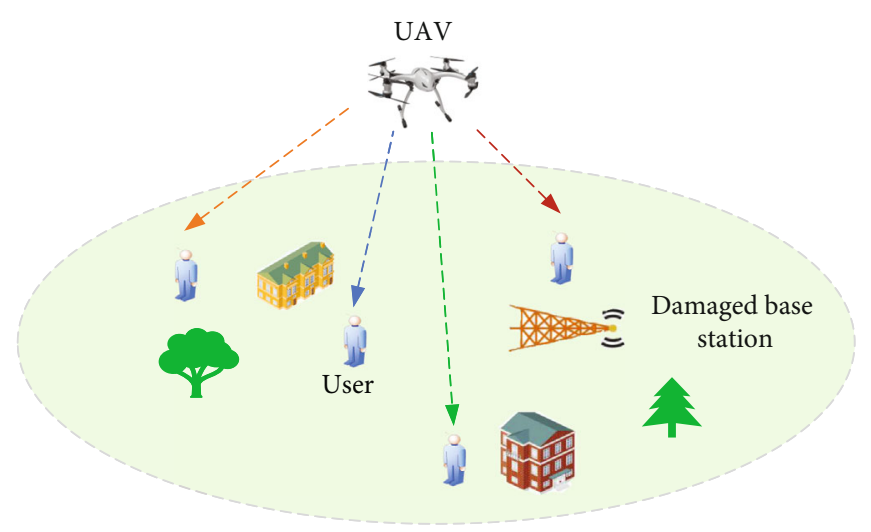

FIgURE 1: A UAV-enabled emergency network.

based A2G propagation to model the large-scale path loss. Then, we formulate an optimization problem to maximize the energy efficiency of the UAV by jointly optimizing the UAV altitude, downlink transmit power, and bandwidth allocation while meeting a statistical delay QoS requirement for each user

(ii) Because the formulated problem is a nonlinear nonconvex optimization problem of fractional programming, it is very difficult to find its optimal solution directly. To this end, we transform the fractional objective function into a tractable subtractive function. Then, we decompose the original optimization problem into three subproblems, i.e., downlink transmit power, bandwidth allocation, and altitude optimization. An efficient iterative algorithm is proposed to obtain the energy efficiency maximization value by using the Dinkelbach method, the block coordinate descent (BCD), and the successive convex optimization (SCO) technique

(iii) Extensive numerical simulations are conducted to prove the performance of our proposed algorithm in three environments. Our simulation results find the optimal altitude for deployment of the UAV in the disaster area. We also extend the optimization of the UAV's location to the 3D space and find the optimal 3D location of the UAV. In addition, we further reveal the characteristics of bandwidth and transmit power allocation in the simulation results. Finally, compared with the benchmark, our algorithm has an obvious performance gain

1.4. Organization. The rest of this paper is organized as follows. In Section 2, we introduce the effective capacity model. System model and the problem formulation for energy efficiency maximization are given in Section 3. In Section 4, an efficient iterative algorithm is proposed by using the Dinkelbach method, the BCD, and the SCO technique. Extensive numerical simulation results are shown in Section 5. In Section 6 , the paper is concluded.

\section{The Theory of Effective Capacity}

In order to describe the statistical QoS provisioning of data transmission in the time-varying wireless channel, we adopted the concept of effective capacity introduced in [9] as the dual concept of effective bandwidth [27]. Specifically, the effective capacity $[9,10,28]$ is defined as the maximum constant arrival rate that can be supported by the service rate $R$ and meets a specific QoS exponent $\theta$. If the service rate $R$ is stationary and time-uncorrelated, the effective capacity can be expressed as follows:

$$
C(\theta)=-\frac{1}{\theta} \ln \left(\mathbb{E}\left\{e^{-\theta R}\right\}\right)(\text { bits })
$$

where $\mathbb{E}\{\cdot\}$ is the expectation operation. Note that the parameter $\theta$, called as the QoS exponent, is a certain positive constant. The parameter $\theta$ is very important for the statistical QoS provisioning, which can characterize the decaying rate of the QoS violation probability. A smaller $\theta$ indicates a slower decay rate, which means that the system can only provide a loose QoS guarantee; on the contrary, a larger $\theta$ represents a faster decay rate, which implies that a more stringent QoS provisioning is required.

The statistical QoS provisioning can be represented as a function of the effective capacity as follows:

$$
\operatorname{Pr}\left\{\text { Delay }>D_{\max }\right\}=e^{-\theta C(\theta) D_{\max }}
$$

where the $D_{\max }$ is the delay-bounding.

\section{System Model and Problem Formulation}

3.1. System Model. As shown in Figure 1, we consider an emergency communication scenario where all terrestrial wireless networks are completely destroyed due to disasters, such as earthquakes and tsunamis. A rotary-wing UAV serving as an aerial base station is employed to provide the multimedia service to $N>1$ ground users, which are denoted by $\mathscr{N}$ with $|\mathcal{N}|=N$, in the downlink. The ground users with different statistical delay QoS requirements are randomly distributed in the disaster area. It is assumed that the UAV base station uses orthogonal frequency division multiple 
access (OFDMA) technology to communicate with ground users in the time horizon $T$.

Without loss of generality, we build a 3D Cartesian coordinate system in which the horizontal coordinate of each ground user $n$ is located at $\mathbf{w}_{n}=\left[x_{n}, y_{n}\right]^{\mathrm{T}} \in \mathbb{R}^{2 \times 1}, n \in \mathcal{N}$. In addition, we assume that the horizontal position of the $\mathrm{UAV}$ is at the origin of coordinate system which is denoted as $(0,0)$. The flying altitude of the UAV is assumed to be $z$, which is greater than the minimum altitude required by local regulations or the minimum safe flying altitude.

The distance between the UAV and the ground user $n$ can be calculated as follows:

$$
d_{n}=\sqrt{x_{n}^{2}+y_{n}^{2}+z^{2}}, \quad \forall n
$$

The air-to-ground (A2G) wireless channel coefficient from the UAV to the ground user $n$ can be expressed as follows:

$$
h_{n}=\sqrt{\chi_{n}} g_{n}, \quad \forall n,
$$

where $\sqrt{\chi_{n}}$ denotes the large-scale average path loss coefficient and $g_{n}$ is the small-scale fading coefficient.

Although the UAV is suspended in the air, due to the complex ground conditions, such as buildings and trees, the A2G link may also be blocked and appear as a non-line-ofsight (NLoS) channel. Therefore, we can model the A2G link as probabilistic LoS and NLoS channel as done in [29-31]. In this model, the A2G path loss is composed of two parts: one part is the large-scale path loss related to distance and the other part is the additional loss based on the LoS probability. The probability that the LoS channel exists between the UAV and the ground user $n$ can be obtained by the following:

$$
P_{\mathrm{LoS}}^{n}=\kappa\left(\frac{180}{\pi} \varphi_{n}-15^{\circ}\right)^{\omega}, \quad \forall n,
$$

where $\kappa$ and $\omega$ are denoted as the parameters, which are related to the geographical environment of signal propagation, and $\varphi_{n}=\arctan ^{-1}\left(z / \sqrt{x_{n}^{2}+y_{n}^{2}}\right)$ is the elevation angle in radian.

Because the probability of the LoS channel and the probability of the NLoS channel sum to 1 , we can obtain the following:

$$
P_{\mathrm{NLoS}}^{n}=1-P_{\mathrm{LoS}}^{n}, \quad \forall n .
$$

For the purpose of simplification, we calculate the expected value of the large-scale path loss, not its random value. The large-scale average path loss can be expressed as follows:

$$
\chi_{n}=\varsigma d_{n}^{-2}\left(P_{\mathrm{LoS}}^{n} 10^{-0.1 \mu_{\mathrm{LoS}}}+P_{\mathrm{NLoS}}^{n} 10^{-0.1 \mu_{\mathrm{NLoS}}}\right), \quad \forall n,
$$

where $\varsigma$ denotes the path loss at the unit distance and $\mu_{\mathrm{LoS}}$ and $\mu_{\mathrm{NLOS}}$ are the additional path losses in $d B$ depending on the LoS condition and the NLoS condition, respectively.
For the purpose of simplification, we can further transform the following equation as follows:

$$
\chi_{n}=d_{n}^{-2}\left(K+L P_{\mathrm{LoS}}^{n}\right), \quad \forall n,
$$

where $K=\varsigma P_{\mathrm{NLoS}}^{n}$ and $L=\varsigma\left(10^{-0.1 \mu_{\mathrm{LoS}}}-10^{-0.1 \mu_{\mathrm{NLOS}}}\right)$.

In addition, we can model the small-scale fading at $\mathrm{A} 2 \mathrm{G}$ channel from the UAV to any ground user $n$ as the Rician fading, because there are usually a dominant LoS component and multipath scatterers. Using this model, $h_{n}$ is the circularly symmetric complex Gaussian distribution $\mathbb{C N}(0,1)$. According to [32], $\left|h_{n}\right|^{2}$ is the small-scale power gain which obeys the noncentral chi-square distribution and is with a normalized average power $E\left[\left|h_{n}\right|^{2}\right]=1$. Thus, the probability distribution function (PDF) of $\left|h_{n}\right|^{2}$ can be written as follows:

$$
\begin{aligned}
f_{\left|h_{n}\right|^{2}}(\omega)= & \frac{\left(K_{n}+1\right) e^{-K_{n}}}{E\left[\left|h_{n}\right|^{2}\right]} \exp \left(\frac{-\left(K_{n}+1\right) \omega}{E\left[\left|h_{n}\right|^{2}\right]}\right) \\
& \times I_{0}\left(2 \sqrt{\frac{K_{n}\left(K_{n}+1\right) \omega}{E\left[\left|h_{n}\right|^{2}\right]}}\right), \quad \omega \geq 0,
\end{aligned}
$$

where $I_{0}$ denotes the zero-order modified Bessel function of the first kind and $k_{n}$ is the Rician factor.

Let $B$ denote the total bandwidth. Because the OFDMA protocol is employed for data transmission, we can divide the total bandwidth into $N$ subcarriers. We assume that the bandwidth allocated to the ground user $n$ is $B_{n}$. So, we have the following:

$$
\sum_{n=1}^{N} B_{n} \leq B
$$

Also, we denote the maximum transmit power as $P . P_{n}$ is the transmit power allocated to the ground user $n$. Therefore, we can obtain the following:

$$
\sum_{n=1}^{N} P_{n} \leq P
$$

We assume that the coherence time of each subcarrier is $T$, which is the frame length of a data transmission. The maximum instantaneous transmission rate for user $n$ in one frame is given by the following:

$$
R_{n}=T B_{n} \log _{2}\left(1+\frac{P_{n} \chi_{n}\left|h_{n}\right|^{2}}{N_{0} B_{n}}\right),
$$

where $N_{0}$ is the single-sided noise spectral density. 
Based on the above derivations, the effective capacity of the ground user $n$ can be obtained by the following:

$$
\begin{aligned}
E_{C}^{n}\left(\left|h_{n}\right|^{2}\right)= & -\frac{1}{\theta_{n}} \ln \mathbb{E}\left(e^{-\theta_{n} T B_{n} \log _{2}\left(1+\left(P_{n} \chi_{n}\left|h_{n}\right|^{2} / N_{0} B_{n}\right)\right)}\right) \\
& =-\frac{1}{\theta_{n}} \ln \int_{0}^{\infty} e^{-\theta_{n} T B_{n} \log _{2}\left(1+\gamma_{n}\right)} f_{\left|h_{n}\right|^{2}}\left(\left|h_{n}\right|^{2}\right) d\left|h_{n}\right|^{2},
\end{aligned}
$$

where $\gamma_{n}=P_{n} \chi_{n}\left|h_{n}\right|^{2} / N_{0} B_{n}$ denotes the signal-to-noise ratio (SNR) of user $n$.

The total power of the UAV mainly includes three parts: data transmission power $P_{T}$, circuit power $P_{C}$, and the UAV hovering power $P_{H}$, which can be written as follows:

$$
P_{t}=\rho P_{T}+P_{C}+P_{H}=\rho \sum_{n=1}^{N} P_{n}+P_{C}+P_{H}
$$

where $1 / \rho$ denotes the drain efficiency of power amplifier.

According to [14], the hovering power of the UAV is related to the altitude, which is given by the following:

$$
P_{H}=\alpha_{H} z+\beta_{H},
$$

where $\alpha_{H}$ and $\beta_{H}$ are the results of curve fitting based on the power/energy values measured in the field experiment.

3.2. Problem Formulation. The UAV energy efficiency, denoted by $\eta_{\mathrm{EE}}$, is defined as the ratio of the sum effective capacity to the energy consumption of the UAV as follows:

$$
\eta_{\mathrm{EE}}=\frac{E_{C}}{P_{t} T}
$$

where $E_{C}$ is the sum effective capacity, which can be expressed as follows:

$$
E_{C}=\sum_{n=1}^{N} E_{C}^{n}
$$

Let $\mathbf{B}=\left\{B_{n}, \forall n\right\}$ and $\mathbf{P}=\left\{P_{n}, \forall n\right\}$. Assuming that the UAV knows the location of each ground user, our goal is to maximize the energy efficiency of the UAV by jointly optimizing the height $z$, power allocation $\mathbf{B}$, and bandwidth allocation $\mathbf{P}$ of the UAV, while meeting a statistical QoS requirement for each user. Mathematically speaking, the optimization problem is formulated as follows:

$$
\begin{gathered}
\max _{z, \mathbf{B}, \mathbf{P}} \frac{\sum_{n=1}^{N}-1 / \theta_{n} \ln \mathbb{E}\left(e^{-\theta_{n} R_{n}}\right)}{\left(\kappa \sum_{n=1}^{N} P_{n}+P_{C}+\alpha_{H} z+\beta_{H}\right) T} \\
\text { s.t. }-\frac{1}{\theta_{n}} \ln \mathbb{E}\left(e^{-\theta_{n} R_{n}}\right) \geq A_{n}, \quad \forall n
\end{gathered}
$$

$$
\begin{gathered}
\sum_{n=1}^{N} B_{n} \leq B \\
B_{n} \geq 0, \quad \forall n \\
\sum_{n=1}^{N} P_{n} \leq P \\
p_{n} \geq 0, \quad \forall n \\
\sum_{n=1}^{N} P_{n}+P_{C}+P_{H} \leq P_{\max } \\
z_{l} \leq z,
\end{gathered}
$$

where the left-hand side (LHS) (Equation (18b)) represents the effective capacity of user $n$ and the right-hand side (RHS) is the minimum effective capacity $A_{n}$ required. In Equation $(18 \mathrm{~g}), P_{\max }$ denotes the total maximum power of the UAV. Besides, $z_{l}$ is the minimum altitude required by local regulations or the minimum safe flying altitude in Equation (18h). Please note that solving Equation (18) is very challenging, mainly due to the following two reasons. First, the objective function is a nonlinear fractional form and is not jointly concave with respect to $\mathbf{B}, \mathbf{P}$, and $z$. Second, Equation (18b) is a nonconvex constraint with respect to $B_{n}, P_{n}$, and $z$. Therefore, Equation (18) is a nonconvex nonlinear fractional form optimization problem which cannot be solved by standard methods efficiently.

\section{Proposed Algorithm}

In this section, Equation (18) is solved in three steps. Specifically, we first convert the fractional form into a subtraction form. However, the transformed objective function is still nonconcave which cannot be also solved by standard methods directly. Then, based on the $\mathrm{BCD}$, the Lagrangian dual method, and the SCO technique, an efficient iterative algorithm is proposed to solve the transformed problem. Finally, the maximal energy efficiency and optimal value of multiple variables are obtained by applying the Dinkelbach method.

4.1. Objective Function Transform. We first convert the fractional form into a subtraction form. Suppose the maximum value of Equation (18) is $\Xi^{*}$, which can meet the equation as follows:

$$
\Xi^{*}=\frac{E_{C}\left(z^{*}, \mathbf{B}^{*}, \mathbf{P}^{*}\right)}{P_{t}\left(z^{*}, \mathbf{B}^{*}, \mathbf{P}^{*}\right) T}=\max _{z, \mathbf{B}, \mathbf{P}} \frac{E_{C}(z, \mathbf{B}, \mathbf{P})}{P_{t}(z, \mathbf{B}, \mathbf{P}) T},
$$

where

$$
E_{C}(z, \mathbf{B}, \mathbf{P})=\sum_{n=1}^{N}-\frac{1}{\theta_{n}} \ln \mathbb{E}\left(e^{-\theta_{n} T B_{n} \log _{2}\left(1+\left(P_{n} \chi_{n}\left|h_{n}\right|^{2} / N_{0} B_{n}\right)\right)}\right),
$$




$$
P_{t}(z, \mathbf{B}, \mathbf{P})=\rho \sum_{n=1}^{N} P_{n}+P_{C}+\alpha_{H} z+\beta_{H},
$$

$\left\{z^{*}, \mathbf{B}^{*}, \mathbf{P}^{*}\right\}$ is the optimal solution of the optimized variable and $\Xi$ is the energy efficiency of Equation (18).

According to [33], the energy efficiency maximization can be obtained, if and only if

$$
\begin{aligned}
& \max _{z, \mathbf{B}, \mathbf{P}} E_{C}(z, \mathbf{B}, \mathbf{P})-\Xi^{*} P_{t}(z, \mathbf{B}, \mathbf{P}) T=E_{C}\left(z^{*}, \mathbf{B}^{*}, \mathbf{P}^{*}\right) \\
& \quad-\Xi^{*} P_{t}\left(z^{*}, \mathbf{B}^{*}, \mathbf{P}^{*}\right) T .
\end{aligned}
$$

Knowing from Equation (22), we can transform the nonlinear fractional objective function of Equation (18) into a subtraction objective function which is a function with variable $\Xi$. Hence, Equation (18) can be rewritten as follows

$$
\begin{gathered}
\max _{z, \mathbf{B}, \mathbf{P}} E_{C}(z, \mathbf{B}, \mathbf{P})-\Xi P_{t}(z, \mathbf{B}, \mathbf{P}) T \\
\text { s.t. }-\frac{1}{\theta_{n}} \ln \mathbb{E}\left(e^{-\theta_{n} R_{n}}\right) \geq A_{n}, \quad \forall n \\
\sum_{n=1}^{N} B_{n} \leq B \\
B_{n} \geq 0, \quad \forall n \\
\sum_{n=1}^{N} P_{n} \leq P \\
p_{n} \geq 0, \quad \forall n \\
\sum_{n=1}^{N} P_{n}+P_{C}+P_{H} \leq P_{\max } \\
z_{l} \leq z .
\end{gathered}
$$

In order to obtain the optimal solution of Equation (23), we propose an efficient iterative algorithm by applying the Dinkelbach method. Furthermore, we show the proposed algorithm in Algorithm 1.

4.2. Optimization for Downlink Transmit Power. For any given UAV altitude $z$ and bandwidth allocation B, Equation (23) can be reformulated as follows:

$$
\begin{gathered}
\max _{\mathbf{P}} E_{C}(z, \mathbf{B}, \mathbf{P})-\Xi P_{t}(z, \mathbf{B}, \mathbf{P}) T \\
\text { s.t. }-\frac{1}{\theta_{n}} \ln \mathbb{E}\left(e^{-\theta_{n} R_{n}}\right) \geq A_{n}, \quad \forall n \\
\sum_{n=1}^{N} P_{n} \leq P \\
P_{n} \geq 0, \quad \forall n \\
\sum_{n=1}^{N} P_{n}+P_{C}+P_{H} \leq P_{\max } .
\end{gathered}
$$

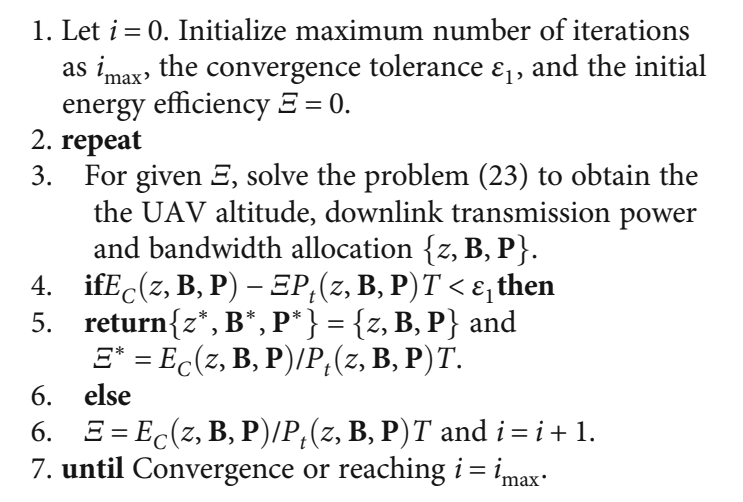

Algorithm 1: Dinkelbach method for Equation (23).

Lemma 1. The objective function $E_{C}(z, B, P)-\Xi P_{t}(z, B, P) T$ is a concave function with respect to $p_{n}$.

Proof. According to Reference [25], $E_{C}(z, B, P)$ is concave with respect to $p_{n}$. Besides, $-\Xi P_{t}(z, B, P) T$ is a linear function respect to $p_{n}$. Since a nonnegative weighted sum of concave functions is concave, the objective function $E_{C}(z, B, P)-\Xi$ $P_{t}(z, B, P) T$ is a concave function with respect to $p_{n}$. Thus, Lemma 1 is fully proved.

According to Lemma 1, Equation (24a) is a concave function with respect to $p_{n}$. The LHS of the constraint (Equation $(22 b))$ is also a concave function with respect to $p_{n}$, which have been proved in [25]. Furthermore, Equations (24c), (24d), and (24e) are all linear constraints. As such, Equation (24) is a convex optimization problem. Furthermore, we can also verify Slater's constraint holds for Equation (24) [34]. According to Slater's theorem, for Equation (24), its strong duality holds and the optimal duality gap is 0 . Therefore, we can solve the dual problem to obtain the optimal solution of Equation (24) by applying the Lagrange duality. The partial Lagrangian of problem (24) can be written as follows:

$$
\begin{aligned}
\mathscr{L}(\mathbf{P}, \lambda, v, \varpi)= & \sum_{n=1}^{N}-\frac{1}{\theta_{n}} \ln \mathbb{E}\left(1+\left(\frac{P_{n} \chi_{n}\left|h_{n}\right|^{2}}{N_{0} B_{n}}\right)^{-\rho}\right) \\
& -\Xi\left(\rho \sum_{n=1}^{N} P_{n}+P_{C}+\alpha_{H} z+\beta_{H}\right) T \\
& +\sum_{n=1}^{N} \lambda_{n}\left(-\frac{1}{\theta_{n}} \ln \mathbb{E}\left(\left(1+\frac{P_{n} \chi_{n}\left|h_{n}\right|^{2}}{N_{0} B_{n}}\right)^{-\rho}\right)-A_{n}\right) \\
& +v\left(P-\sum_{n=1}^{N} P_{n}\right)+\bowtie\left(P_{\max }-\sum_{n=1}^{N} P_{n}-P_{C}-P_{H}\right)
\end{aligned}
$$

where $\mathbf{B}=\left\{B_{n}, \forall n\right\}, \mathbf{P}=\left\{P_{n}, \forall n\right\}$, and $\rho=\theta T B_{n} / \ln 2$. Moreover, $\lambda, v$, and $\omega$ denote the Lagrange multiplier vectors associated with the constraints (Equations (24b), (24c), and (24e)), which lie in Equation (24). Constraint (Equation (24d)) will be used when we consider the boundary of the optimal solution in the following. 
In order to facilitate the subsequent calculations, we transform Lagrangian (Equation (25)) into the form as follows:

$$
\mathscr{L}(\mathbf{P}, \lambda, v, \varpi)=\sum_{n=1}^{N} Y+\Gamma
$$

where

$$
\begin{aligned}
Y=\left(\lambda_{n}+1\right)\left(-\frac{1}{\theta_{n}} \ln \mathbb{E}\left(\left(1+\frac{P_{n} \chi_{n}\left|h_{n}\right|^{2}}{N_{0} B_{n}}\right)^{-\rho}\right)\right) \\
-(\Xi \rho+\omega+v) P_{n}, \\
\Gamma=-\Xi\left(P_{C}+\alpha_{H} z+\beta_{H}\right) T+\sum_{n=1}^{N} \lambda_{n}\left(-A_{n}\right) \\
+v P+\omega\left(P_{\max }-P_{C}-P_{H}\right) .
\end{aligned}
$$

For the given Lagrange multiplier vectors $(\lambda, v, \varpi)$, Equation (26) can be regarded as $N$ parallel subproblems, which can be solved independently. Because Equation (28) is not related to $\mathbf{B}$ and $\mathbf{P}$, it can be considered as a constant. Therefore, when we solve the optimal solution of Equation (24), Equation (28) can be ignored. Let $P_{n}^{*}$ denote the optimal solution of Equation (27), when Equation (27) obtains the maximum value. Due to the concave characteristic of Equation (27) with respect to $P_{n}$, we can solve it by applying the Karush-Kuhn-Tucker (KKT) conditions. Next, we take the partial derivatives of Equation (27) with respect to $P_{n}$ as follows:

When the constraint (Equation (24d)) is considered, the optimal solution, denoted by $P_{n}^{*}$, can meet the following:

$$
\frac{\partial Y}{\partial P_{n}}=\left(1+\lambda_{n}\right) \frac{\rho\left(\chi_{n}\left|h_{n}\right|^{2} / N_{0} B_{n}\right)\left(1+\left(P_{n}^{*} \chi_{n}\left|h_{n}\right|^{2} / N_{0} B_{n}\right)\right)^{-\rho-1}}{\theta \mathbb{E}\left(\left(1+\left(P_{n}^{*} \chi_{n}\left|h_{n}\right|^{2} / N_{0} B_{n}\right)\right)^{-\rho}\right)}-(\Xi \rho+\omega+v),
$$

$$
\begin{cases}\left.\frac{\partial Y}{\partial P_{n}}\right|_{P_{n}=0}<0, & P_{n}^{*}=0, \\ \left.\frac{\partial Y}{\partial P_{n}}\right|_{P_{n}=0 P_{n}^{*}}, & P_{n}^{*}>0 .\end{cases}
$$

Then, the optimal solution for the power allocated $P_{n}$ to user $n$ can be obtained as follows:

$$
P_{n u}^{*}=\left[\frac{\rho^{1 /(1+\rho)}}{\Theta^{1 /(1+\rho)}\left(\chi_{n}\left|h_{n}\right|^{2} / N_{0} B_{n}\right)^{\rho /(1+\rho)}}-\frac{N_{0} B_{n}}{\chi_{n}\left|h_{n}\right|^{2}}\right]^{+},
$$

where $\Theta=(\Xi \rho+\emptyset+v) \theta \mathbb{E}\left(\left(1+\left(P_{n}^{*} \chi_{n}\left|h_{n}\right|^{2} / N_{0} B_{n}\right)\right)^{-\rho}\right) / 1+$ $\lambda_{n}$ and $[x]^{+}=\max \{0, x\}$. Furthermore, according to a similar lemma in [28], we know that the value of $\Theta$ can be obtained when the constraint is satisfied with equality. Therefore, by inserting Equation (31) into Equation (24b) and changing the inequality operation with equality, we have the following:

$$
-\frac{1}{\theta_{n}} \ln \mathbb{E}\left(\left(1+\frac{\rho^{1 /(1+\rho)} / \Theta^{1 /(1+\rho)}\left(\chi_{n}\left|h_{n}\right|^{2} / N_{0} B_{n}\right)^{\rho /(1+\rho)}-\left(N_{0} B_{n} / \chi_{n}\left|h_{n}\right|^{2}\right) \chi_{n}\left|h_{n}\right|^{2}}{N_{0} B_{n}}\right)^{-\rho}\right)=0,
$$

where the expectation operations can be processed by applying the closed-form expressions in [35]. When $\Theta$ is found, we can solve Equation (31) to obtain the optimum power allocation $P_{n}^{*}$.

4.3. Optimization for Bandwidth Allocation. For any given UAV altitude $z$ and power allocation B, Equation (23) can be solved by optimizing the following problem:

$$
\begin{gathered}
\max _{\mathbf{B}} E_{C}(z, \mathbf{B}, \mathbf{P})-\Xi P_{t}(z, \mathbf{B}, \mathbf{P}) T \\
\text { s.t. }-\frac{1}{\theta_{n}} \ln \mathbb{E}\left(e^{-\theta_{n} R_{n}}\right) \geq A_{n}, \quad \forall n \\
\sum_{n=1}^{N} B_{n} \leq B,
\end{gathered}
$$

where the first term of the objective function $E_{C}(z, \mathbf{B}, \mathbf{P})$ is a concave function with respect to $\mathbf{B}$, which has been proved in [25]. In addition, constraint (Equation (33a)) has also been proven to be a concave function with respect to $B_{n}$. Finally, constraint (Equation (33c)) is a linear function. So, Equation (33) is a standard convex optimization problem which can be solved by applying the interior-point method [34].

4.4. Optimization for the UAV's Altitude. With given any power and bandwidth allocation $\{\mathbf{B}, \mathbf{P}\}$, Equation (23) can be transformed as follows:

$$
\begin{gathered}
\max _{z} E_{C}(z, \mathbf{B}, \mathbf{P})-\Xi P_{t}(z, \mathbf{B}, \mathbf{P}) T \\
\text { s.t. }-\frac{1}{\theta_{n}} \ln \mathbb{E}\left(e^{-\theta_{n} T B_{n} \log _{2}\left(1+\left(P_{n} \chi_{n}\left|h_{n}\right|^{2} / N_{0} B_{n}\right)\right)}\right) \geq A_{n}, \quad \forall n
\end{gathered}
$$

$$
z_{l} \leq z,
$$

where the objective function of Equation (34) is nonconcave with respect to $z$. At the same time, constraint (Equation (34b)) is a nonconcave constraint. Only, constraint (Equation (34c)) is a linear constraint. Thus, Equation (34) is a nonconvex problem. In general, there is no efficient way to solve this type of problem. Next, successive convex optimization technique will be used to obtain the suboptimal solution for bandwidth allocation B. To this end, by introducing slack variables $\iota=\left\{\iota_{n}=K\right.$ $\left.+L \kappa\left(180 / \pi \arctan ^{-1}\left(z / \sqrt{x_{n}^{2}+y_{n}^{2}}\right)-15^{\circ}\right)^{\omega} / x_{n}^{2}+y_{n}^{2}+z^{2}, \forall n\right\}$, Equation (34) can be rewritten as follows:

$$
\max _{z} \sum_{n=1}^{N}-\frac{1}{\theta_{n}} \ln \mathbb{E}\left(e^{-\theta_{n} R_{n 1}}\right)-\Xi T\left(\alpha_{H} z+\sqsubseteq\right)
$$




$$
\begin{gathered}
\text { s.t. }-\frac{1}{\theta_{n}} \ln \mathbb{E}\left(e^{-\theta_{n} R_{n 1}}\right) \geq A_{n}, \quad \forall n \\
\frac{K+L \kappa\left(180 / \pi \arctan ^{-1}\left(z / \sqrt{x_{n}^{2}+y_{n}^{2}}\right)-15^{\circ}\right)^{\omega}}{x_{n}^{2}+y_{n}^{2}+z^{2}} \geq \iota_{n}, \quad \forall n \\
z_{l} \leq z
\end{gathered}
$$

where

$$
\begin{gathered}
R_{n 1}=T B_{n} \log _{2}\left(1+\frac{P_{n} \iota_{n}\left|h_{n}\right|^{2}}{N_{0} B_{n}}\right), \quad \forall n, \\
\sqsubseteq=\rho \sum_{n=1}^{N} P_{n}+P_{C}+\beta_{H} .
\end{gathered}
$$

The LHS of constraint (Equation (33b)) is a concave function with respect to $z$ which has been proved in [25]. Since the first term of the objective function (Equation (35a)) is a nonnegative weighted sum of the LHS constraint (Equation (35b)), it is concave. Then, the first term of the objective function (Equation (35a)) subtracts $\sqsubseteq$, which is a linear function with respect to $z$. Therefore, the objective function (Equation (35a)) is a concave function with respect to $z$. However, constraint (Equation (35c)) is still a nonconvex constraint. To deal with the nonconvexity of Equation (35c), we introduce slack variables $\xi=\left\{\xi_{n}=K+L \kappa\right.$ $\left.\left(180 / \pi \arctan ^{-1}\left(z / \sqrt{x_{n}^{2}+y_{n}^{2}}\right)-15^{\circ}\right)^{\omega}, \forall n\right\}$ and $\boldsymbol{\Omega}=\left\{\Omega_{n}=x_{n}^{2}\right.$ $\left.+y_{n}^{2}+z^{2}, \forall n\right\}$. So, Equation (35) can be reformulated as follows:

$$
\begin{gathered}
\max _{z} \sum_{n=1}^{N}-\frac{1}{\theta_{n}} \ln \mathbb{E}\left(e^{-\theta_{n} R_{n 1}}\right)-\Xi T\left(\alpha_{H} z+\sqsubseteq\right) \\
\text { s.t. }-\frac{1}{\theta_{n}} \ln \mathbb{E}\left(e^{-\theta_{n} R_{n 1}}\right) \geq A_{n}, \quad \forall n \\
\frac{\xi_{n}}{\Omega_{n}} \geq \iota_{n}, \quad \forall n \\
K+L \kappa\left(\frac{180}{\pi} \arctan ^{-1}\left(\frac{z}{\sqrt{x_{n}^{2}+y_{n}^{2}}}\right)-15^{\circ}\right)^{\omega} \geq \xi_{n}, \quad \forall n \\
x_{n}^{2}+y_{n}^{2}+z^{2} \leq \Omega_{n}, \quad \forall n \\
z_{l} \leq z
\end{gathered}
$$

where according to [29], the LHS of constraint (Equation (38d)) is a concave function. Since the LHS of constraint (Equation (38d)) is a second-order cone, constraint (Equation (38d)) is a convex constraint. Unfortunately, constraint (Equation (38c)) is still a nonconvex constraint. In the following, taking natural logarithms on both sides of constraint (Equation (38c)), we
1. Let $l=0$. Initialize $\iota_{n}^{0}$ and $\Omega_{n}^{0}$.

2. repeat

3. Solve the problem (42) to obtain the optimal $z^{*}$. Let $z^{l+1}=z^{*}$.

4. Update $l=l+1$.

5. until Convergence or reaching the maximum number of iterations.

6. Output $z^{*}$.

Algorithm 2: Proposed algorithm for Equation (34) via SCO.

have the following:

$$
\ln \xi_{n} \geq \ln \iota_{n}+\ln \Omega_{n}, \quad \forall n .
$$

To tackle the nonconvexity of Equation (39), we use the successive convex optimization technique to approximate $\ln t_{n}$ in each iteration. Based on the characteristics of concave functions, we know that the first-order Taylor expansion of any concave function is its global upper estimate. Therefore, given $l_{n}^{l}$ as value of $\iota_{n}$ in the $l$-th iteration, we have the following:

$$
\ln \iota_{n} \leq \ln l_{n}^{l}+\frac{1}{l_{n}^{l}}\left(l_{n}^{l}-\iota_{n}\right), \quad \forall n .
$$

Similarly, we have the following:

$$
\ln \Omega_{n} \leq \ln \Omega_{n}^{l}+\frac{1}{\Omega_{n}^{l}}\left(\Omega_{n}^{l}-\Omega_{n}\right), \quad \forall n,
$$

where $\Omega_{n}^{l}$ is the value of $\Omega_{n}$ in the $l$-th iteration.

Applying the SCO technique, Equation (38) is approximated as the following problem:

$$
\begin{gathered}
\max _{z, \xi, \Omega, l} \sum_{n=1}^{N}-\frac{1}{\theta_{n}} \ln \mathbb{E}\left(e^{-\theta_{n} R_{n 1}}\right)-\Xi T\left(\alpha_{H} z+\sqsubseteq\right) \\
\text { s.t. }-\frac{1}{\theta_{n}} \ln \mathbb{E}\left(e^{-\theta_{n} R_{n 1}}\right) \geq A_{n}, \quad \forall n \\
\ln \xi_{n} \geq \ln l_{n}^{l}+\frac{1}{l_{n}^{l}}\left(l_{n}^{l}-\iota_{n}\right)+\ln \Omega_{n}^{l}+\frac{1}{\Omega_{n}^{l}}\left(\Omega_{n}^{l}-\Omega_{n}\right), \quad \forall n \\
K+L \kappa\left(\frac{180}{\pi} \arctan ^{-1}\left(\frac{z}{\sqrt{x_{n}^{2}+y_{n}^{2}}}\right)-15^{\circ}\right)^{\omega} \geq \xi_{n}, \quad \forall n \\
x_{n}^{2}+y_{n}^{2}+z^{2} \leq \Omega_{n}, \quad \forall n
\end{gathered}
$$

Now, Equation (42) has been a convex optimization problem, which can be solved by applying the interior- 


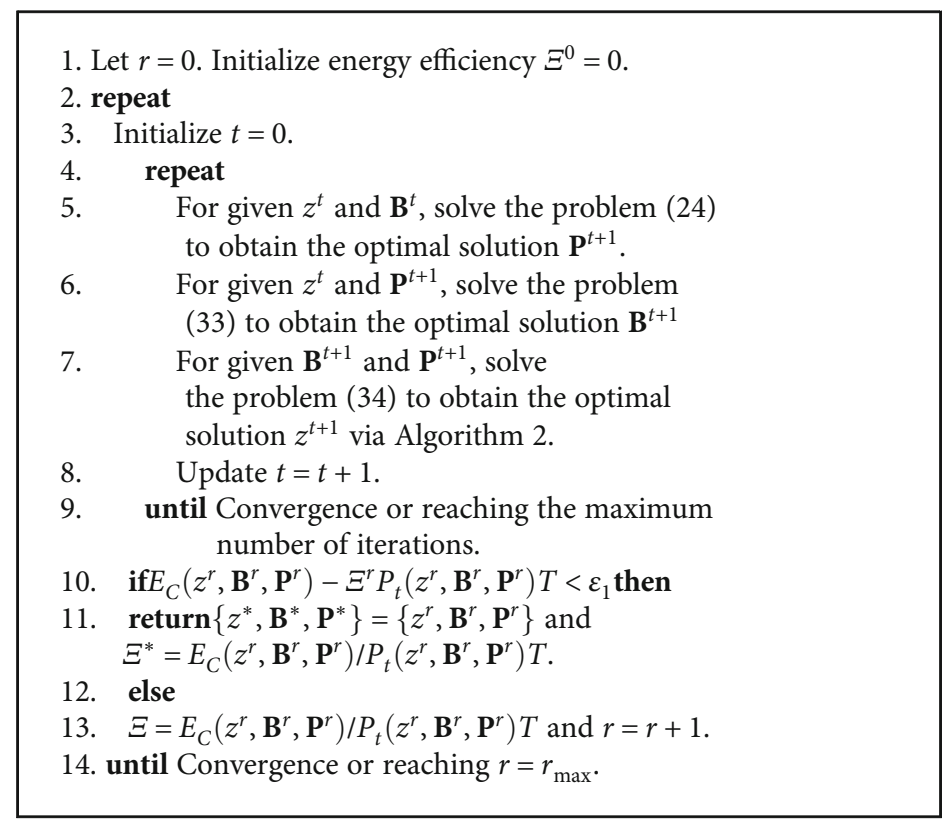

Algorithm 3: Overall iterative optimization algorithm for Equation (18).

point method [34]. The proposed algorithm for obtaining the optimal UAV altitude by applying the SCO technique is summarized in Algorithm 2.

4.5. Overall Algorithm Design. To solve Equation (18), we propose an overall iterative optimization algorithm with a double-loop structure, which has been summarized in Algorithm 3. The outer loop in Algorithm 3 is used to calculate energy efficiency $\Xi$ through the Dinkelbach method that has been summarized in Algorithm 1. The inner loop uses the block coordinate descent method to alternately iteratively optimize the UAV altitude, power, and bandwidth allocation to obtain the optimal solution when $\Xi^{r}$ is given in the outer loop. Due to the UAV altitude solved by SCO, we can only get the suboptimal solution of Equation (18).

4.6. Computational Complexity Analysis. In this subsection, the computational complexity of Algorithm 3 is analyzed in detail. Based on Algorithm 1 and Algorithm 2, we can find the proposed iterative optimization (Algorithm 3) has a double-loop structure. Specifically, the outer loop obtains the energy efficiency $\Xi$ based on the Dinkelbach method, which is executed a total of $r_{\max }$ iterations. The inner loop based on the BCD algorithm is iterated for a total of $t_{\max }$ in the worst case when the outer loop is executed each time. The computational complexity of the inner loop consists of three parts. The first part is for obtaining the optimal solution of Equation (24), whose computational complexity is $O(N)$ in step 5 of Algorithm 3 . In the second part, the computational complexity of solving Equation (33) is $O\left(N^{3.5}\right)$ by applying the interiorpoint method in step 6 of Algorithm 3 [34]. We solve Equation (34) to obtain the optimal solution via Algorithm 2 whose computational complexity is $O\left(l_{\max }(3 N+1)^{3.5}\right)$ [36] in the
TABLE 1: Coordinates of the three users.

\begin{tabular}{lc}
\hline & Coordinates \\
\hline User 1 & $(-402.4,-221.5,0)$ \\
User 2 & $(46.8,457.5,0)$ \\
User 3 & $(464.8,-342.3,0)$ \\
\hline
\end{tabular}

TABLE 2: User parameters.

\begin{tabular}{lcc}
\hline & $A_{n}($ bits $)$ & $\theta_{n}$ \\
\hline User 1 & 100 & $10^{-2}$ \\
User 2 & 200 & $10^{-3}$ \\
User 3 & 300 & $10^{-4}$ \\
\hline
\end{tabular}

TABle 3: Environmental parameters.

\begin{tabular}{lccc}
\hline & Suburban & Urban & Dense urban \\
\hline$\left(\mu_{\mathrm{LoS}}, \mu_{\mathrm{NLoS}}\right)$ & $(0.1,21)$ & $(1,20)$ & $(1.6,23)$ \\
$(\kappa, \omega)$ & $(0.76,0.06)$ & $(0.6,0.11)$ & $(0.36,0.21)$ \\
\hline
\end{tabular}

third part. Therefore, the overall computational complexity of Algorithm 3 is $O\left(r_{\max }\left(t_{\max }\left(N+N^{3.5}+l_{\max }(3 N+1)^{3.5}\right)\right)\right)$, i.e., $O\left(r_{\max }\left(t_{\max }\left(N^{3.5}+l_{\max }(3 N)^{3.5}\right)\right)\right)$.

\section{Numerical Results}

In this section, numerical results are shown to validate the proposed algorithm. Without loss of generality, we consider that $N=3$ ground users are randomly distributed in the disaster area of $1 \mathrm{~km} \times 1 \mathrm{~km}$, whose center coordinate is $(0$, $0,0)$. We use MATLAB to randomly generate the 


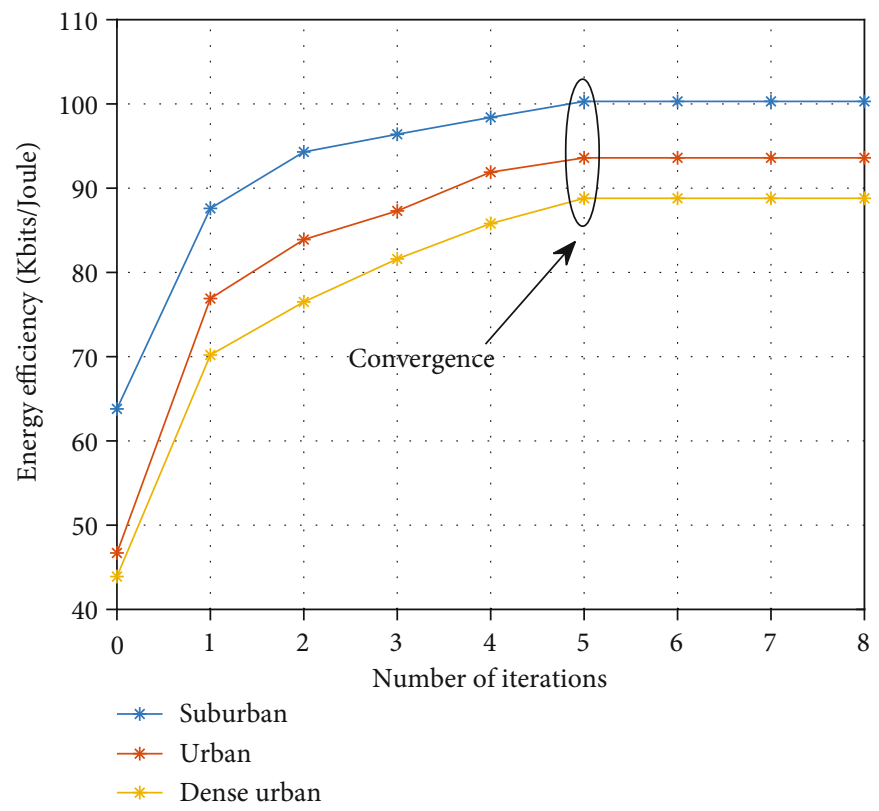

Figure 2: Convergence of the proposed Algorithm 3 for three environments.

coordinates of three ground users, which are given in Table 1. Furthermore, we set these three ground users to have different QoS exponent and minimum effective capacity requirements, which are given in Table 2 . In addition, we consider the numerical results to be simulated in the suburban, urban, and dense urban environments, respectively. According to [29], we list the detailed environmental parameters in Table 3. For the parameters related to UAV power, we set $P$ $=1 \mathrm{~W}$ and $P_{C}=5 \mathrm{~W}, \alpha_{H}=4.917$ and $\beta_{H}=275.204$ [14]. We assume that the minimum hovering altitude $z_{l}$ of the $\mathrm{UAV}$ is $200 \mathrm{~m}$. Besides, we set $K_{n}=4, \varepsilon_{1}=10^{-3}, r_{\max }=50, \varsigma$ $=-40 \mathrm{~dB}, B=5 \mathrm{MHz}, N_{0}=-174 \mathrm{dBm} / \mathrm{Hz}$, and $T=0.02 \mathrm{~ms}$. The above parameters are used in our simulations unless otherwise stated. In order to evaluate the performance of our proposed algorithm, we design a benchmark, in which we consider equal resource allocation $\left\{B_{1}=B_{2}=B_{3}=1 / 3 B, P_{1}\right.$ $\left.=\mathrm{P}_{2}=P_{3}=1 / 3 P\right\}$, while the UAV is deployed at the minimum allowed altitude $z=z_{l}=200 \mathrm{~m}$.

In Figure 2, we present the convergence behavior of proposed Algorithm 3 for three different environments. It is worth noting that we are mainly concerned with the number of iterations in the outer loop rather than the inner loop, because energy efficiency $\Xi$ is updated only in each iteration of the Dinkelbach method. First, we can find that our proposed algorithm can quickly converge in three environments after 5 iterations. Therefore, our proposed Algorithm 3 is proved to have a very good convergence rate. Second, we can observe that the highest energy efficiency is in the suburban environment. This is because there is the highest probability of the LoS link $P_{\text {LoS }}^{n}$ between the UAV and the ground users in the suburban environment, which can be calculated from Equation (5).

In Figure 3, we show the optimal altitude at which the $\mathrm{UAV}$ is deployed in three environments. By using Algo-

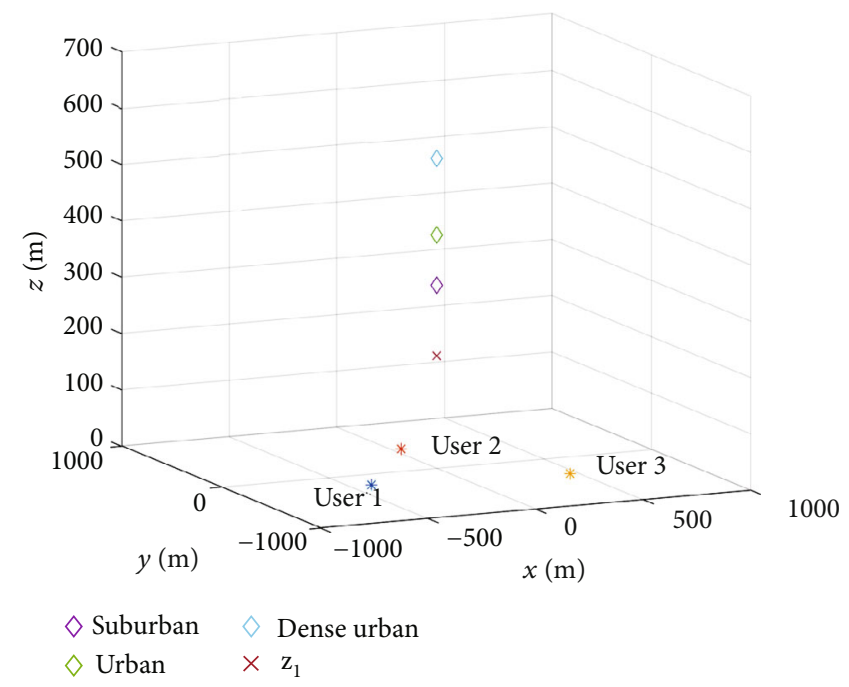

FIgURE 3: The optimal altitude of UAV for three environments.

rithm 3, we find that the optimal deployment altitudes of the UAV are $325.3 \mathrm{~m}, 413.6 \mathrm{~m}$, and $549.2 \mathrm{~m}$ in the suburban, urban, and dense urban environments, respectively. According to Equation (15), as the altitude of the UAV increases, the hovering energy consumption of the UAV increases. However, from Figure 3, we observe that the $\mathrm{UAV}$ does not hover at the minimum allowed altitude $z_{l}$ $=200 \mathrm{~m}$ in the three environments. This is because the UAV obtains a higher probability of LoS by increasing the hovering altitude, thereby improving the channel gain and increasing the sum effective capacity of ground users. In addition, we can also see that the hover altitude of the $\mathrm{UAV}$ is the highest in the dense urban environment, 


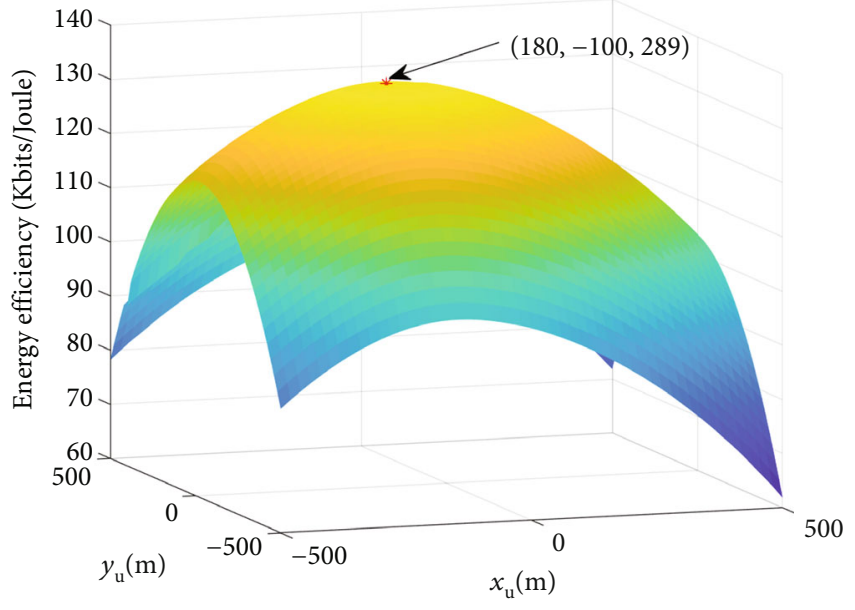

(a) Suburban

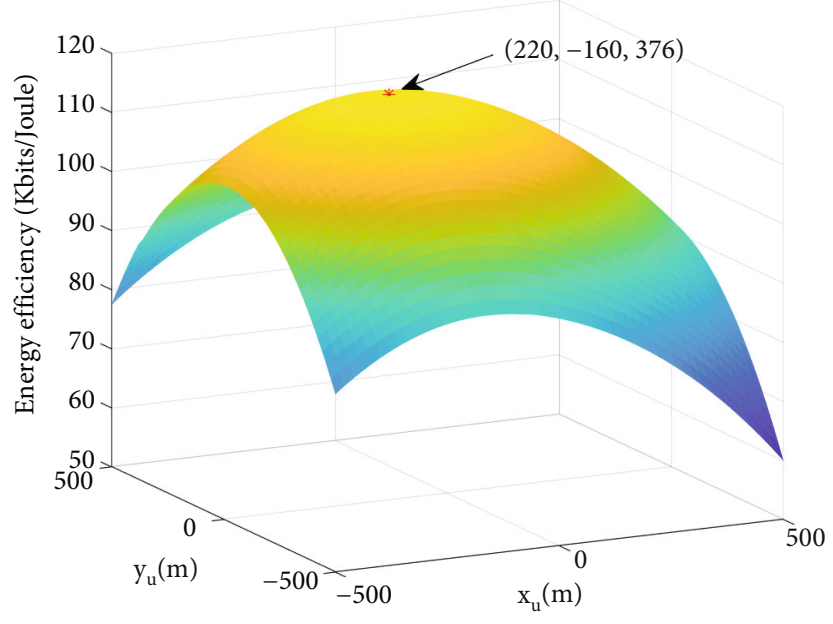

(b) Urban

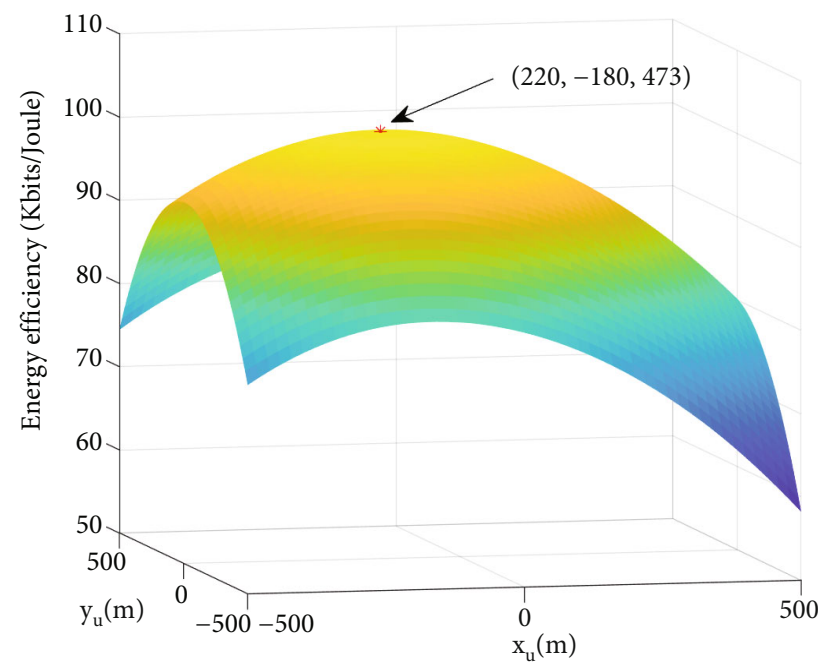

(c) Dense urban

FIGURE 4: The optimal 3D UAV location for three environments.

because the dense urban environment has the worst channel propagation environment. In Figure 4, we give the optimal 3D position of the UAV, which is obtained by numerical simulations. We can find that the horizontal position of the UAV is closer to the user 3 in the three environments. The reason for this is that the QoS exponent of user 3 is the smallest. When the UAV is closer to user 3, it can obtain a larger effective capacity and achieve the higher energy efficiency.

Figure 5 shows the energy efficiency versus the maximum transmit power $P$ in three environments. When given any $P$, we can obtain the maximum energy efficiency by Algorithm 3. We can find from Figure 5 that when the maximum transmit power $P$ is the same in the three environments, the maximum energy efficiency is always the highest in the suburban environment. This is expected since the channel quality is best in the suburban environment, so according to Equation (12), where the Shannon channel capacity is maximum when the transmit power is equal. According to the effective capacity Equation (13), we know that the Shannon capacity and the effective capacity are positively correlated, and thus, the effective capacity is also the largest in the suburban environment. In addition, we know from Figure 3 that the hovering altitude of the UAV in the suburban environment is the lowest among the three environments. According to Equation (15), the hovering energy consumption in the suburban environment is the least, so the energy efficiency is the largest in the suburban environment. Compared with the benchmark, the energy efficiency increment is the largest in the dense urban environment. This shows that our proposed Algorithm 3 improves energy efficiency more obviously for environments with poorer channel quality.

In Figure 6, we show the optimal power allocation of the three users versus the maximum transmit power $P$ in the urban environment. $P_{1}, P_{2}$, and $P_{3}$ represent the optimal power allocated to user 1 , user 2 , and user 3 , respectively. 


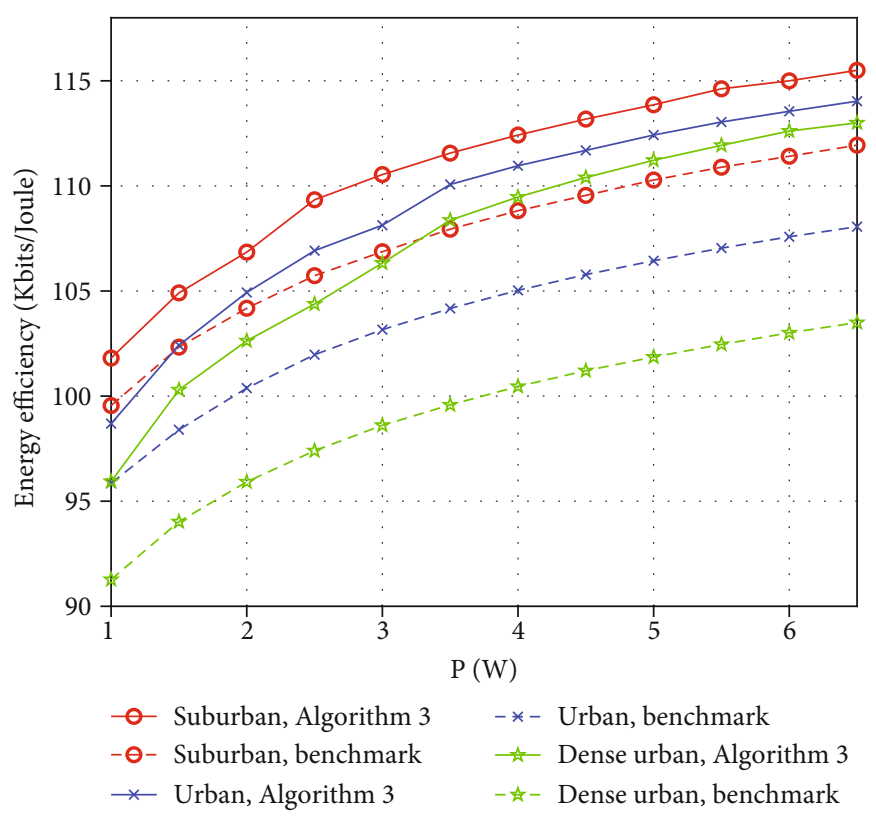

FIgURe 5: Energy efficiency versus the maximum transmit power $P$.

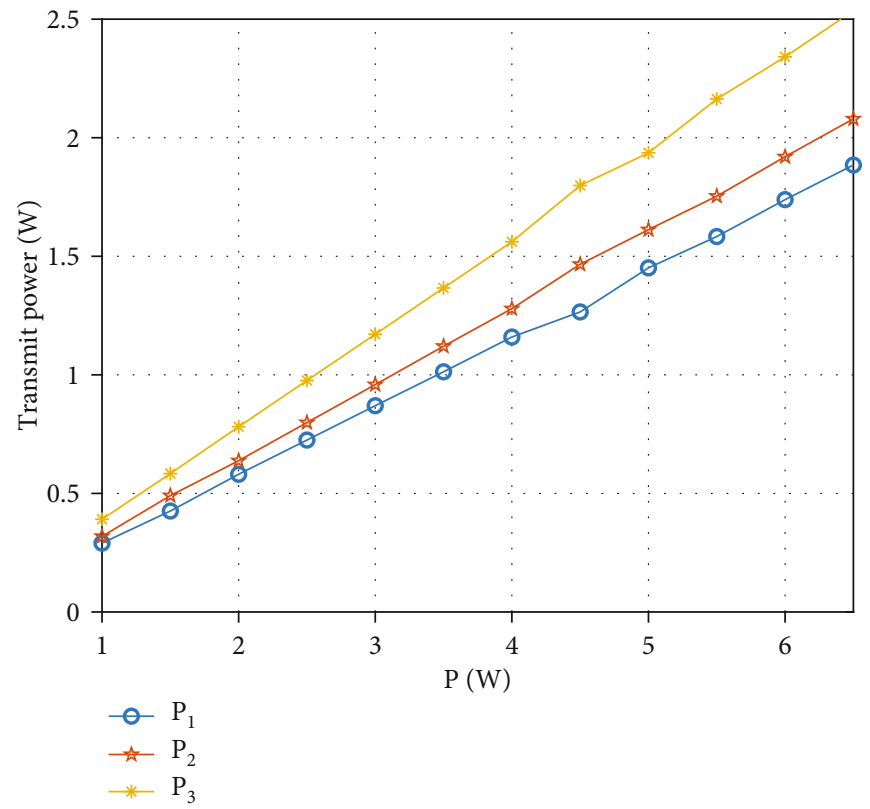

Figure 6: The optimal power allocation of the three users versus the maximum transmit power $P$ in an urban environment.

From Figure 6, we can observe that when $P$ is relatively small, $P_{1}$ and $P_{2}$ are almost equal and the difference with $P_{3}$ is very small. The reason is that user 1 and user 2 have larger QoS exponent $\left\{\theta_{1}, \theta_{2}\right\}$. To provide a more stringent QoS provisioning, from Equation (1), the higher transmission power needs to be provided. Furthermore, we can get that as $P$ increases, the difference between $P_{3}$ and the other two $P_{1}$ and $P_{2}$ becomes larger and larger. It can be interpreted that user 3 has the smallest $Q o S$ exponent $\theta_{3}$, and as $P$ increases, the greater the power allocated to user 3, the more the effective capacity increases, so the maximum energy efficiency is obtained. Similarly, Figure 7 presents the characteristics of optimal bandwidth allocation in a suburban environment. We see that the bandwidth $B_{3}$ allocated to user 3 has always been the largest. It is expected since user 3 has the smallest QoS exponent. The more bandwidth is allocated to user 3, the more effective capacity is increased and therefore the greater the energy efficiency. 


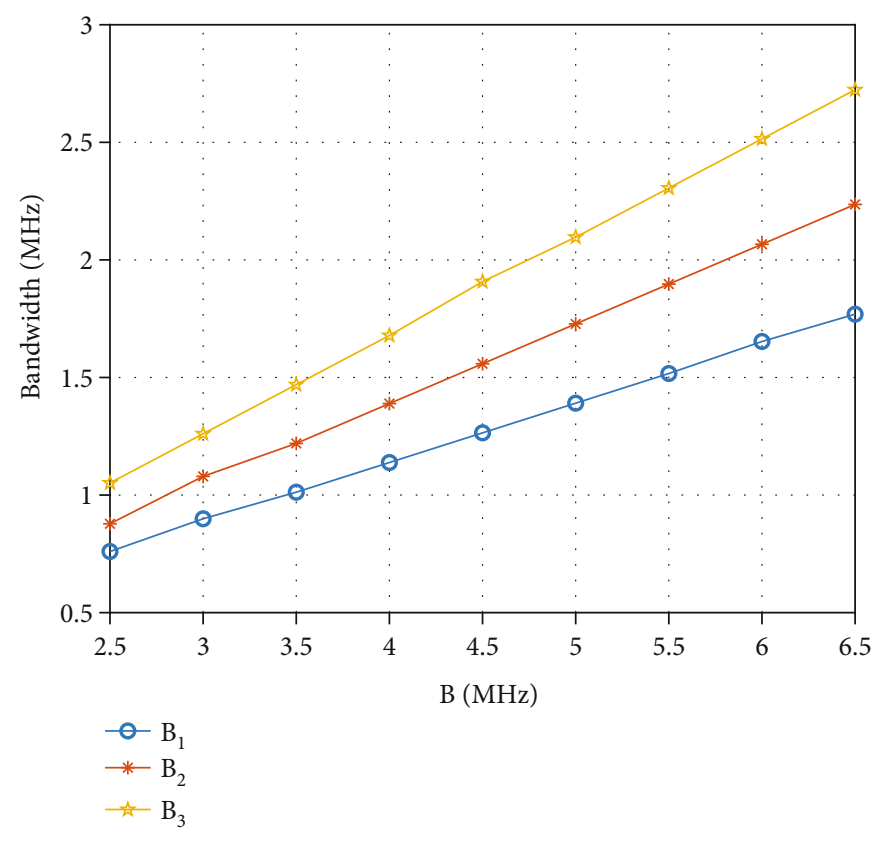

Figure 7: Bandwidth allocation versus $B$ in the suburban environment.

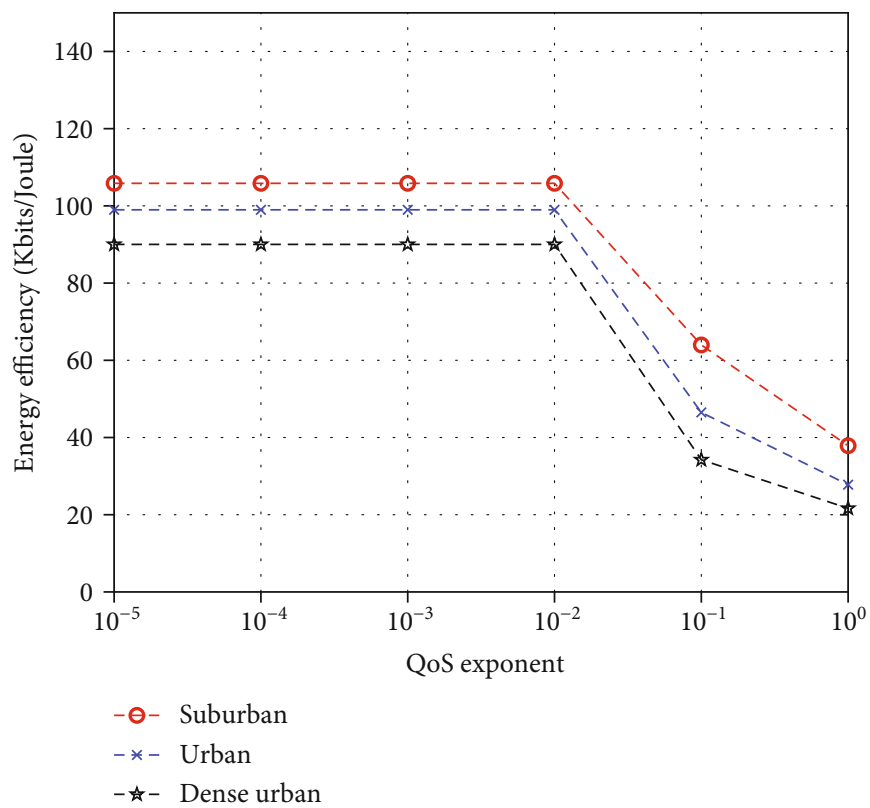

FIGURE 8: Energy efficiency versus the QoS exponent.

To further clarify the relationship between QoS exponent and energy efficiency, we assume $\theta_{1}=\theta_{2}=\theta_{3}$ and $A_{1}=A_{2}$ $=A_{3}$. In Figure 8, we plot the energy efficiency versus the QoS exponent. From Figure 8, we can find that when the QoS exponent is relatively small, the energy efficiency is almost unchanged as the QoS exponent increases. It can be interpreted as when the QoS exponent is small, the delay requirement is relatively loose, and the effective capacity is close to the Shannon capacity; that is to say, the effective capacity is almost unchanged with the QoS exponent. Therefore, the energy efficiency is almost unchanged. However, as the QoS exponent becomes larger, starting from $10^{-2}$, the energy efficiency begins to decline rapidly. This is because when the QoS exponent is larger, more power is used to the minimum effective capacity requirement $A_{n}$, which can be explained by Equation (13).

In Figure 9, we compare the energy efficiency obtained by our proposed Algorithm 3 with the energy efficiency 


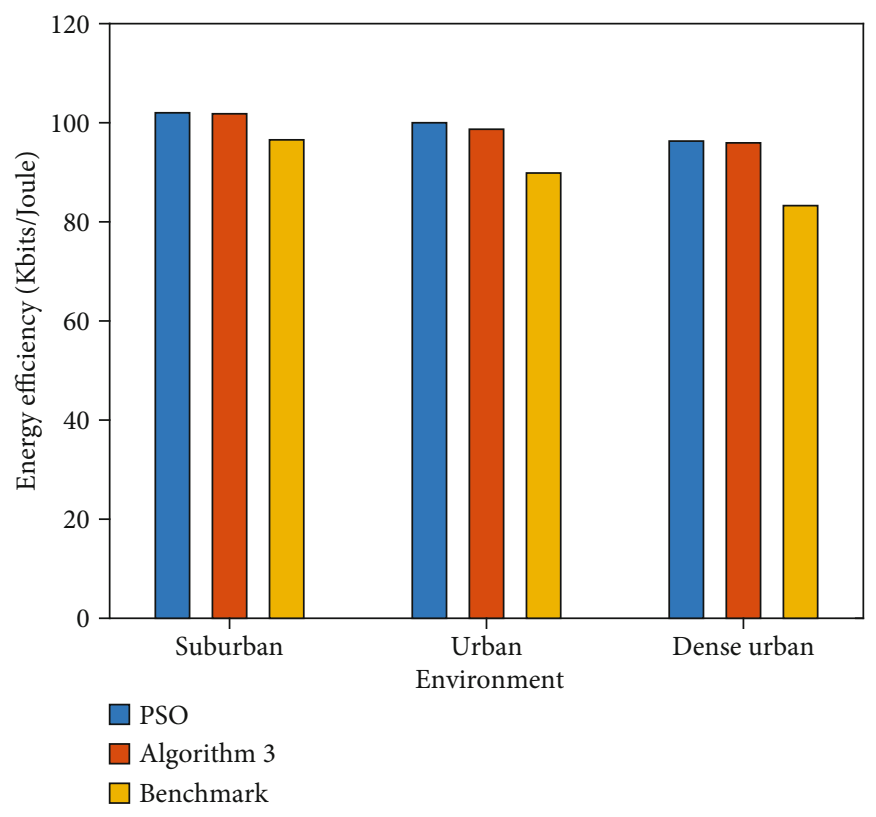

FIgURE 9: The maximum energy efficiency of UAV for three environments.

obtained by the particle swarm optimization (PSO) algorithm and the benchmark. We can find that the energy efficiency of our proposed Algorithm 3 is significantly higher than that of the benchmark. At the same time, we can also see that the energy efficiency of our proposed Algorithm 3 is almost equal to that obtained by PSO.

\section{Conclusion}

In this paper, we have investigated an energy-efficient UAV emergency network, where a UAV works as an aerial base station to serve a group of users with different statistical QoS constraints in the downlink. Then, we have formulated an optimization problem to maximize the energy efficiency of the UAV by jointly optimizing the UAV altitude, downlink transmission power, and bandwidth allocation while meeting a statistical delay QoS requirement for each user. Consider that the formulated optimization problem is a nonlinear nonconvex optimization problem of fractional programming that is challenging to solve. In order to deal with the nonconvex optimization problem, we have applied the Dinkelbach method, the BCD, and the SCO technique to propose an efficient iteration algorithm. Extensive simulation results have verified that our proposed algorithm has fast convergence characteristics and a significant performance gain compared with a benchmark.

\section{Data Availability}

No data were used to support this study.

\section{Conflicts of Interest}

The authors declare that they have no conflicts of interest.

\section{References}

[1] N. Zhao, W. Lu, M. Sheng et al., "UAV-assisted emergency networks in disasters," IEEE Wireless Communications, vol. 26, no. 1, pp. 45-51, 2019.

[2] Y. Zeng, R. Zhang, and T. J. Lim, "Wireless communications with unmanned aerial vehicles: opportunities and challenges," IEEE Communications Magazine, vol. 54, no. 5, pp. 36-42, 2016.

[3] C. Zhang, L. Zhang, L. Zhu, T. Zhang, Z. Xiao, and X.-G. Xia, "3D deployment of multiple UAV-mounted base stations for UAV communications," IEEE Transactions on Communications, vol. 69, no. 4, pp. 2473-2488, 2021.

[4] T. Bai, C. Pan, J. Wang et al., "Dynamic aerial base station placement for minimum-delay communications," IEEE Internet of Things Journal, vol. 8, no. 3, pp. 1623-1635, 2021.

[5] X. Jing, J. Sun, and C. Masouros, "Energy aware trajectory optimization for aerial base stations," IEEE Transactions on Communications, vol. 69, no. 5, pp. 3352-3366, 2021.

[6] Z. Sheng, H. D. Tuan, T. Q. Duong, and L. Hanzo, "UAVaided two-way multi-user relaying," IEEE Transactions on Communications, vol. 69, no. 1, pp. 246-260, 2021.

[7] B. Yang, T. Taleb, Y. Fan, and S. Shen, "Mode selection and cooperative jamming for covert communication in D2D underlaid UAV networks," IEEE Network, pp. 1-8, 2021.

[8] G. Forecast, "Cisco visual networking index: global mobile data traffic forecast update, 2017-2022," Update, vol. 2017, p. 2022, 2019.

[9] D. Wu and R. Negi, "Effective capacity: a wireless link model for support of quality of service," IEEE Transactions on Wireless Communications, vol. 2, no. 4, pp. 630-643, 2003.

[10] M. Amjad, L. Musavian, and M. H. Rehmani, "Effective capacity in wireless networks: a comprehensive survey," IEEE Communications Surveys \& Tutorials, vol. 21, no. 4, pp. 3007-3038, 2019. 
[11] W. Cheng, X. Zhang, and H. Zhang, "Statistical-QoS driven energy efficiency optimization over green $5 \mathrm{G}$ mobile wireless networks," IEEE Journal on Selected Areas in Communications, vol. 34, no. 12, pp. 3092-3107, 2016.

[12] X. Zhang, W. Cheng, and H. Zhang, "Heterogeneous statistical QoS provisioning over airborne mobile wireless networks," IEEE Journal on Selected Areas in Communications, vol. 36, no. 9, pp. 2139-2152, 2018.

[13] N. Babu, K. Ntougias, C. B. Papadias, and P. Popovski, "Energy efficient altitude optimization of an aerial access point," in 2020 IEEE 31st Annual International Symposium on Personal, Indoor and Mobile Radio Communications, pp. 1-7, 2020.

[14] H. V. Abeywickrama, B. A. Jayawickrama, Y. He, and E. Dutkiewicz, "Comprehensive energy consumption model for unmanned aerial vehicles, based on empirical studies of battery performance," IEEE Access, vol. 6, pp. 58 383-58 394, 2018.

[15] L. Wang, B. Hu, and S. Chen, "Energy efficient placement of a drone base station for minimum required transmit power," IEEE Wireless Communications Letters, vol. 9, no. 12, pp. 2010-2014, 2020.

[16] S. C. Noh, H. B. Jeon, and C. B. Chae, "Energy-efficient deployment of multiple UAVs using ellipse clustering to establish base stations," IEEE Wireless Communications Letters, vol. 9, no. 8, pp. 1155-1159, 2020.

[17] K. M. S. Huq, S. Mumtaz, Z. Zhou, K. Chandra, I. E. Otung, and J. Rodriguez, "Energy-efficiency maximization for D2Denabled UAV aided 5G networks," in ICC 2020-2020 IEEE International Conference on Communications (ICC), pp. 1-6, 2020.

[18] Y. Zeng and R. Zhang, "Energy-efficient UAV communication with trajectory optimization," IEEE Transactions on Wireless Communications, vol. 16, no. 6, pp. 3747-3760, 2017.

[19] Y. Zeng, J. Xu, and R. Zhang, "Energy minimization for wireless communication with rotary-wing UAV," IEEE Transactions on Wireless Communications, vol. 18, no. 4, pp. 23292345, 2019.

[20] B. Duo, Q. Wu, X. Yuan, and R. Zhang, "Energy efficiency maximization for full-duplex UAV secrecy communication," IEEE Transactions on Vehicular Technology, vol. 69, no. 4, pp. 4590-4595, 2020.

[21] M. Hua, Y. Wang, C. Li, Y. Huang, and L. Yang, "Energy-efficient optimization for UAV-aided cellular offloading," IEEE Wireless Communications Letters, vol. 8, no. 3, pp. 769-772, 2019.

[22] Y. Huang, M. Cui, G. Zhang, and W. Chen, "Bandwidth, power and trajectory optimization for UAV base station networks with backhaul and user QoS constraints," IEEE Access, vol. 8, pp. 67 625-67 634, 2020.

[23] V. Sharma, R. Sabatini, and S. Ramasamy, "UAVs assisted delay optimization in heterogeneous wireless networks," IEEE Communications Letters, vol. 20, no. 12, pp. 2526-2529, 2016.

[24] M. Z. Hassan, M. J. Hossain, J. Cheng, and V. C. M. Leung, "Statistical-QoS guarantee for IoT network driven by laserpowered UAV relay and RF backscatter communications," IEEE Transactions on Green Communications and Networking, vol. 5, no. 1, pp. 406-425, 2021.

[25] H. Niu, X. Zhao, and J. Li, "3D location and resource allocation optimization for UAV-enabled emergency networks under statistical QoS constraint," IEEE Access, vol. 9, pp. 41 566-41 576, 2021.
[26] S. Zhang and W. Cheng, "Statistical QoS provisioning for UAV-enabled emergency communication networks," 2019 IEEE Globecom Workshops (GC Wkshps), pp. , 20191-6, 2019.

[27] C.-S. Chang and J. A. Thomas, "Effective bandwidth in highspeed digital networks," IEEE Journal on Selected Areas in Communications, vol. 13, no. 6, pp. 1091-1100, 1995.

[28] L. Musavian and Q. Ni, "Effective capacity maximization with statistical delay and effective energy efficiency requirements," IEEE Transactions on Wireless Communications, vol. 14, no. 7, pp. 3824-3835, 2015.

[29] L. Wang, B. Hu, F. Wang, S. Chen, and J. Cui, "Joint altitude, power control, and bandwidth allocation optimization for unmanned aerial vehicle-enabled reliable communications," Transactions on Emerging Telecommunications Technologies, vol. 31, no. 7, article e3983, 2020.

[30] A. Al-Hourani, S. Kandeepan, and A. Jamalipour, "Modeling air-to-ground path loss for low altitude platforms in urban environments," in 2014 IEEE Global Communications Conference. IEEE, pp. 2898-2904, 2014.

[31] A. Al-Hourani, S. Kandeepan, and S. Lardner, "Optimal lap altitude for maximum coverage," IEEE Wireless Communications Letters, vol. 3, no. 6, pp. 569-572, 2014.

[32] M. M. Azari, F. Rosas, K.-C. Chen, and S. Pollin, "Ultra reliable UAV communication using altitude and cooperation diversity," IEEE Transactions on Communications, vol. 66, no. 1, pp. 330-344, 2017.

[33] W. Dinkelbach, "On nonlinear fractional programming," Management Science, vol. 13, no. 7, pp. 492-498, 1967.

[34] S. Boyd, S. P. Boyd, and L. Vandenberghe, Convex Optimization, Cambridge University Press, 2013.

[35] A. J. Goldsmith and P. P. Varaiya, "Capacity of fading channels with channel side information," IEEE Transactions on Information Theory, vol. 43, no. 6, pp. 1986-1992, 1997.

[36] Q. Wu and R. Zhang, "Common throughput maximization in UAV-enabled OFDMA systems with delay consideration," IEEE Transactions on Communications, vol. 66, no. 12, pp. 6614-6627, 2018. 